\title{
Minimization of spin tune spread for preservation of spin polarization at RHIC
}

\author{
C. Liu, J. Kewisch, H. Huang, and M. Minty \\ Brookhaven National Laboratory, Upton, New York 11973, USA
}

(Received 10 December 2018; published 18 June 2019)

\begin{abstract}
The spin structure of the proton is one of the unsolved problems in physics. The Relativistic Heavy Ion Collider (RHIC) Spin Physics Program has been conducting research to investigate the spin structure by colliding high energy polarized proton beams. Maintaining high spin polarization while achieving high luminosity is critical for this program. Two of the challenges related to the program are to maintain spin polarization during spin flipping, a process designed to reduce systematic errors in spin asymmetry measurements, and during beam acceleration from the low energy source to the high energy collision experiment. Recently, a modification of the accelerator's magnet configuration (optics) was developed for reducing the spin tune spread. With the new optics implemented, higher spin flipping efficiency was achieved at both injection and top beam energies. The spin tune spread was also minimized during beam acceleration and the transmission efficiency of spin polarization was evaluated. In this report the modifications needed for minimizing the spin tune spread and their impact on the global beam optics are described.
\end{abstract}

DOI: 10.1103/PhysRevAccelBeams.22.061002

\section{INTRODUCTION}

Preserving spin polarization during beam acceleration through the accelerator chain and during spin flipping in RHIC are two substantial challenges for the RHIC Spin Physics Program [1,2]. Although spin preservation has been improved in the injectors, especially in the alternating gradient synchrotron (AGS) with multiple measures to counteract the depolarizing resonances, the proton beam loses significant polarization along the transport from the proton source to the collision of high energy protons at RHIC. It was observed that part of the polarization loss is caused by the strong intrinsic resonances during beam acceleration in RHIC [2,3] despite the presence of Siberian snakes. In addition, despite upgrades of the spin flipper system [4-8] and improvement of the related closed orbit bump, spin polarization was not well preserved during spin flipping experiments until the optics was modified for reducing the spin tune spread. As described in this report the modification consists in minimizing the difference of the dispersion derivatives (dispersion primes) between the two Siberian snakes [9] used for polarization preservation. With the new optics the spin polarization was reported [10]

*cliu1@bnl.gov

Published by the American Physical Society under the terms of the Creative Commons Attribution 4.0 International license. Further distribution of this work must maintain attribution to the author(s) and the published article's title, journal citation, and DOI. to be well preserved during spin flipping. The approach was similarly applied in an attempt to improve the preservation of spin polarization during beam acceleration in RHIC.

This article describes the optics modifications to reduce the spin tune spread including simulations and experimental results. The benefits of a narrower spin tune spread were theoretically predicted [11,12], but the optics modification for reducing the spin tune spread proposed in the past either has not been tested due to power supply limit [13] or has not been implemented successfully [14]. This work demonstrated for the first time reduction of the spin tune spread by optics modification in RHIC.

This paper is structured as follows. In Sec. II, the RHIC polarized proton operation, the RHIC spin flipper and the motivation for minimizing the spin tune spread are introduced. Section III describes the dispersion prime matching implemented in RHIC. Section IV presents the experimental results at fixed energies after the new optics was implemented. Section V presents the experimental results during beam acceleration after the same matching scheme was applied at intermediate energy points. Summary and outlooks for future work are given in Sec. VI.

\section{BACKGROUND AND MOTIVATION FOR MINIMIZING SPIN TUNE SPREAD}

\section{A. Polarization preservation during beam transport in RHIC}

The spin structure of proton $[1,2]$ is studied in RHIC with collisions of high-energy polarized proton beams. The 
spin polarized proton beam is generated by an optically pumped polarized ion source (OPPIS) [15], accelerated in the linac, the booster, the AGS and then injected into either of the two RHIC rings (denoted as "blue" and "yellow") [16]. During beam acceleration, the spin polarization may not be preserved due to depolarizing resonances $[3,17,18]$. Depolarizing resonances occur when the spin tune $\nu_{s p}$, which denotes the number of spin rotations about the stable spin direction in one revolution of the beam in the accelerator, satisfies the resonance condition,

$$
\begin{aligned}
\nu_{s p} & =G \gamma \\
& = \begin{cases}k P+n, & \text { Imperfection resonances } \\
k P \pm l \nu_{x} \pm m \nu_{y} \pm n \nu_{z}, & \text { Intrinsic resonances }\end{cases}
\end{aligned}
$$

where $G=1.7928$ is the anomalous magnetic moment of the proton, $\gamma$ is the relativistic Lorentz factor, $P$ is the superperiodicity of the accelerator, $\nu_{x}$ is the horizontal betatron tune, $\nu_{y}$ is the vertical betatron tune, $\nu_{z}$ is the synchrotron tune, and $k, l, m$, and $n$ are integers.

Over the course of many years, various measures were taken to improve the polarization transmission efficiency through the pre-accelerators and RHIC. In the booster, depolarizing resonances are mitigated using harmonic orbit correction $[19,20]$ for the imperfection resonances. In the AGS, two partial Siberian snakes [21-24] are used to reduce the impact of both imperfection and vertical intrinsic resonances and a pair of horizontal tune jump quadrupoles are applied to overcome horizontal intrinsic resonances [25-27]. In RHIC, two Siberian snakes [9,28] were placed on opposite sides of each of the two RHIC rings in 2001. Each Siberian snake consists of a sequence of superconducting helical dipole magnets which rotate the spin by 180 degrees per passage. The Siberian snakes force the spin tune to be $1 / 2$ independent of beam energy thus avoiding the imperfection and intrinsic depolarizing resonance conditions.

\section{B. Introduction to the RHIC spin flipper and the motivation for minimizing spin tune spread}

By sweeping the frequency of an rf solenoid across a depolarizing resonance, significant spin flipping efficiency has been achieved for proton beams [29,30]. Since the spin rotation due to the solenoid's magnetic field integral decreases linearly with beam momentum, use of a solenoid is impractical for flipping the spin of high energy beams $[31,32]$. Therefore, an rf dipole spin flipper was proposed [4] and designed for RHIC.

The spin flipper [4] was installed in one of the RHIC rings (Blue) to demonstrate flipping the spin of high energy protons. The spin flipper consists of four horizontal DC dipoles and five vertical AC dipoles [6-8]. The spin flipper driving tune, i.e., the ratio of the spin flipper driving frequency to the beam revolution frequency, sweeps adiabatically across the spin tune to flip the spin direction by 180 degrees [29].

Spin polarization preservation during spin flipping depends strongly on the spin tune spread. To first order, in the presence of two diametrically opposed Siberian snakes, the spin tune is expressed as [11],

$$
\nu_{s p}=\frac{1}{2}+\frac{1+G \gamma}{\pi}\left(D_{1}^{\prime}-D_{2}^{\prime}\right) \frac{\Delta p}{p},
$$

where $D_{1}^{\prime}$ and $D_{2}^{\prime}$ (dispersion prime) are the derivatives of the dispersion functions with respect to the longitudinal coordinate at each of the two Siberian snakes respectively, $p$ is the beam momentum and $\Delta p$ is the beam momentum spread. The absolute value of the second term,

$$
\delta \nu_{s p}=\frac{1+G \gamma}{\pi}\left|D_{1}^{\prime}-D_{2}^{\prime}\right| \frac{\Delta p}{p},
$$

is the spin tune spread. Momentum spread of the beam results in nonzero spin tune spread due to the difference of the dispersion primes at the snakes. As a result, the spin flipper driving tune crosses the spin tune of off-energy particles multiple times (Fig. 1) when flipping the spin direction [33] resulting in a significant loss of spin polarization. A narrower spin tune spread therefore reduces the possibility of multiple crossings. Consequently, higher spin flipping efficiency can be achieved as explored in simulations $[14,34]$. The spin tune spread is proportional to the difference of the dispersion prime, $D_{1}^{\prime}-D_{2}^{\prime}$, at the two

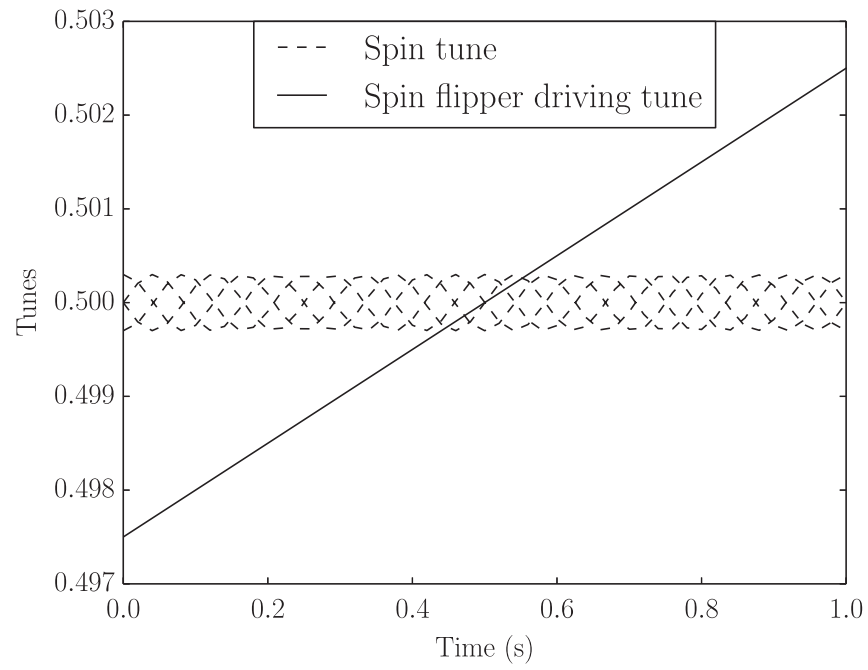

FIG. 1. Mechanism of multiple spin tune crossings by the spin flipper driving tune at injection energy. The spin tunes for offenergy particles (dashed curves for three different phases) oscillate with the synchrotron frequency $(\sim 6 \mathrm{~Hz})$. The spin flipper tune (the solid line) is ramped across the design spin tune $\left(\nu_{\mathrm{sp}}=1 / 2\right)$ and intersects the spin tune of some of the particles multiple times. 

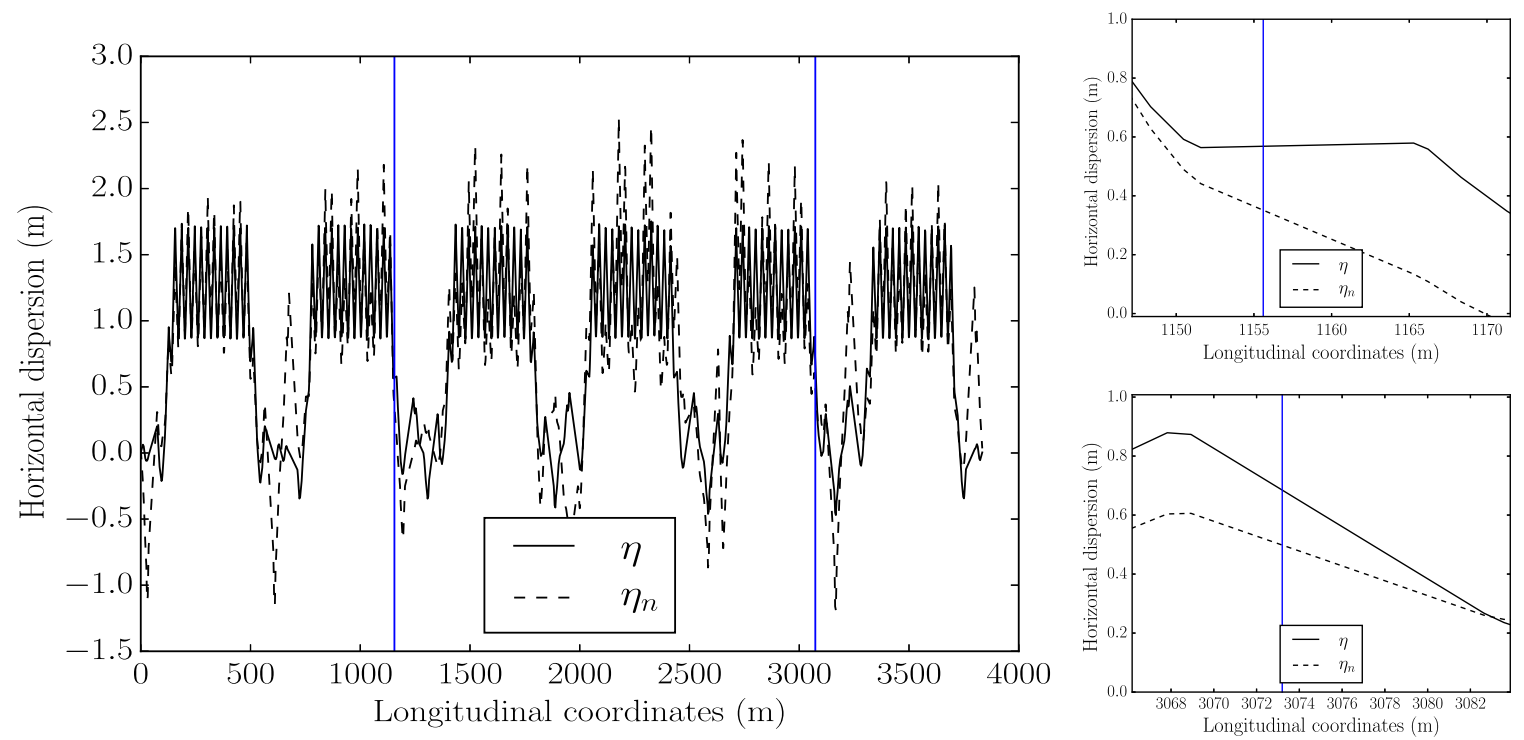

FIG. 2. Left plot: The model horizontal dispersion functions (the solid line) at top energy and the distorted horizontal dispersion functions (the dashed line) with the dispersion primes matched at the two Siberian snakes (located at the vertical lines) in the Blue ring. Right plots: Expanded view of the dispersion functions at the first snake (the upper plot) and the second snake (the lower plot). The lattice was designed for the 2017 polarized proton program at top energy.

Siberian snakes; therefore, the spin tune spread can be reduced by matching the dispersion primes at the two Siberian snakes.

\section{MATCHING DISPERSION PRIME AT THE SIBERIAN SNAKES}

The $\gamma_{T}$ quadrupoles, designed to facilitate fast crossing of transition energy by quickly changing the transition gamma, were re-purposed to match the dispersion primes at the two Siberian snakes. There are $24 \gamma_{T}$ quadrupoles per accelerator with 4 in each of the 6 arcs sharing a common power supply. In standard operation $\left(\gamma_{T}\right.$ jump configuration), the first and the third $\gamma_{T}$ quadrupoles in the same arc generate a closed dispersion bump, as do the second and the fourth $\gamma_{T}$ quadrupoles. Meanwhile, the relative $\beta$-function changes ( $\beta$-beat) introduced by each pair of adjacent $\gamma_{T}$ quadrupoles cancel each other. In this configuration the $\gamma_{T}$ quadrupoles are not able to adjust the dispersion and dispersion prime at the Siberian snakes. When the polarities of the second and third quadrupole are reversed, these $\gamma_{T}$ quadrupoles generate a perturbation to the dispersion function which propagates all around the accelerator. The tune change and the $\beta$-beat introduced by the $\gamma_{T}$ quadrupoles are minor due to cancellation of the individual contributions. Tune jump quadrupoles, of which two are located at the end of each arc where the dispersion function is close to zero, are used to keep the betatron tunes constant during the $\gamma_{T}$ jump. These are also used to compensate for minor tune changes caused by the $\gamma_{T}$ quadrupoles in the matching of dispersion prime.
The dispersion prime matching solution, e.g., the polarities and strengths of the $\gamma_{T}$ quadrupoles, was determined by using MAD-X [35] matching module. With the $\gamma_{T}$ quadrupoles treated as magnets with individual power supplies, the strength of each quadrupole magnet was first determined by asking the dispersion primes at the two Siberian snakes to be equal. The $\gamma_{T}$ quadrupoles in the same arc were assigned with proper polarities. The matching was repeated by adding the condition of constant betatron tunes.

The resulting perturbation to the optical functions has been evaluated and results are here presented for the blue ring lattice at top energy $(254.8 \mathrm{GeV})$ used in the 2017 polarized proton program [36]. The perturbation to the dispersion function due to the matching is shown in Fig. 2. The dispersion functions around the snakes are shown in the expanded view on the right. The slopes of the curves crossing the snakes represent the dispersion primes at the snakes $\left(D_{1}^{\prime}\right.$ is for the snake at $1155.7 \mathrm{~m}, D_{2}^{\prime}$ is for the snake at $3073.2 \mathrm{~m}$ ). The exact value of $\delta D^{\prime}=D_{1}^{\prime}-D_{2}^{\prime}$ is listed in Table I and will be compared with measurement in Sec. IV C. The dispersion at the collision point was constrained to be zero to avoid possible beam-beam induced synchrobetatron coupling [37]. The resulting relative change of the $\beta$-functions is shown in Fig. 3.

The dispersion primes at the Siberian snakes were also matched for 20 energy points along beam acceleration including injection and top energy. The perturbation to the dispersion function due to the matching was found to be of similar magnitude at all beam energies, an example corresponding to top energy is shown in Fig. 2. 
TABLE I. Comparison of $\delta D^{\prime}$ from model prediction and measurements.

\begin{tabular}{lccrr}
\hline \hline Energy $(\mathrm{GeV})$ & $\delta D_{\text {model }}^{\prime}$ without & $\delta D_{\text {model }}^{\prime}$ with & $\delta D_{\text {measurement without }}^{\prime}$ & $\delta D_{\text {measurement }}^{\prime}$ with \\
$D^{\prime}$-matching & $D^{\prime}$-matching & $D^{\prime}$-matching & $-0.0035 \pm 0.0002$ \\
\hline 23.8 & -0.0606 & 0 & $-0.0740 \pm 0.0001$ & $-0.0001 \pm 0.0002$ \\
254.8 & -0.0453 & 0 & $-0.0557 \pm 0.0001$ & - -matching \\
\hline \hline
\end{tabular}

The perturbation to the $\beta$-functions at all energies was found to be smaller than that at top energy (Fig. 3). The standard deviation of the relative changes of the $\beta$-functions at all the beam energies during beam acceleration is shown in Fig. 4.

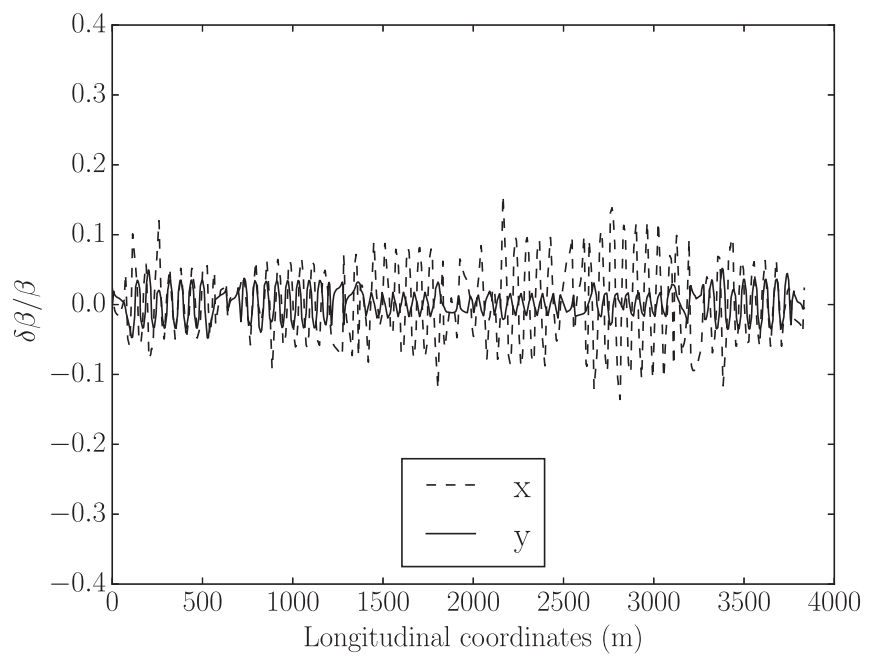

FIG. 3. The resulting relative changes of the $\beta$-functions at top energy in the Blue ring, with the dispersion primes matched at the two Siberian snakes. The horizontal values are denoted by the dashed line and the vertical ones by the solid line.

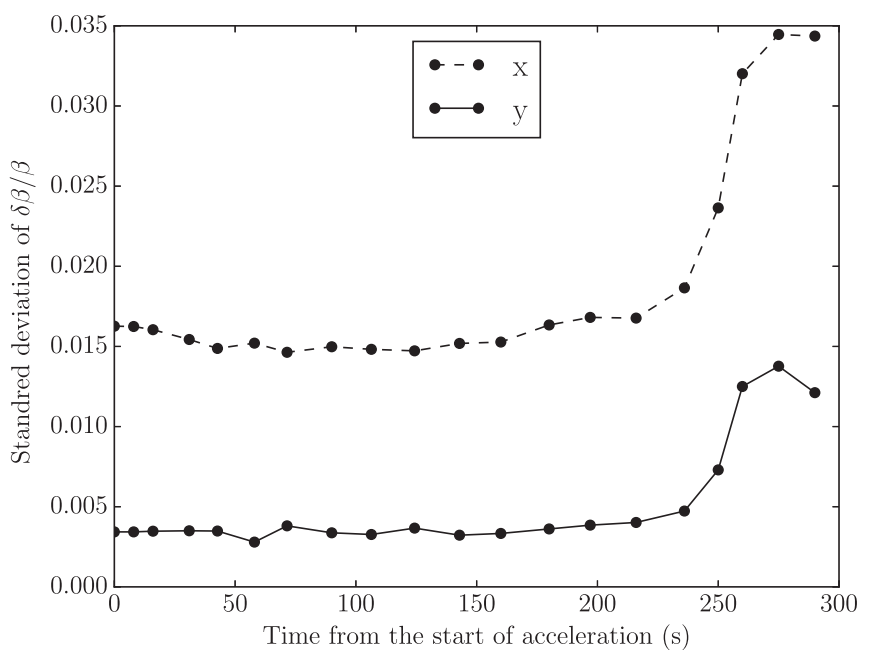

FIG. 4. The standard deviation of the resultant relative changes of the $\beta$-functions during the 280 second beam acceleration cycle with the dispersion primes matched at the two Siberian snakes in the blue ring.

\section{IMPLEMENTATION OF DISPERSION PRIME MATCHING AT FIXED ENERGIES AND EXPERIMENTAL RESULTS}

\section{A. Experimental results at injection energy}

The dispersion prime matching solution was implemented in the blue ring at injection energy $(23.8 \mathrm{GeV})$. The measured dispersion function before matching and after matching at injection energy are shown in Fig. 5. The dispersion primes at the Siberian snakes were calculated as the ratio between the difference of the measured dispersions at the entrance and exit beam position monitors (BPMs) and the distance between the BPMs $(\delta s=13.11 \mathrm{~m})$,

$$
\delta D^{\prime}=\frac{D_{1}-D_{2}}{\delta s}
$$

The initial difference of dispersion prime at the two Siberian snakes was measured to be $\delta D^{\prime}=-0.0740 \pm$ 0.0001 at injection energy. It was reduced to $\delta D^{\prime}=$ $-0.0035 \pm 0.0002$ after implementing the matched dispersion primes optics. The errors represent statistical errors derived from four sets of dispersion measurements.

The spin flipping experiments were performed at injection energy with dispersion primes matched by sweeping the spin flipper's tune across a 0.005 range over a certain time period (sweep time). The spin tune spread was measured to be a factor of $\sim 20$ narrower with the matching of the dispersion primes at the two Siberian snakes [10]. A spin flipping efficiency of $\sim 92 \%$ was achieved with the reduced spin tune spread and a sweep time of three seconds [10]. The spin flipping efficiency was improved to $~ 97 \%$ with a sweep time of one second [10] compared to 50\% achieved without $\delta D^{\prime}$-matching in the past [8].

\section{B. Experimental results at top energy}

The dispersion prime matching solution was implemented in subsequent experiments at top energy. The residual difference of the dispersion primes was measured to be $\delta D^{\prime}=-0.0030 \pm 0.0001$. The spin of the protons was flipped with an efficiency of about $70 \%$. The dispersion prime difference was further reduced to $\delta D^{\prime}=$ $-0.0001 \pm 0.0002$ by introducing a dispersion prime difference at the two Siberian snakes equal and opposite to the measured residual. After this reduction of the dispersion prime difference, the spin flipping efficiency was improved to $\sim 90 \%$ and $\sim 97 \%$ with a sweep time of one and one-half second respectively [10]. The measured 



FIG. 5. Left plot: The measured horizontal dispersion functions in the blue ring at the beam position monitors at injection energy before (the solid line) and after (the dashed line) matching of the dispersion primes at the two Siberian snakes (located at the vertical lines). Right plots: Expanded view of the dispersion functions at the first snake (the upper plot) and the second snake (the lower plot).

dispersion function after the dispersion prime matching at top energy is shown in Fig. 6 and is compared with the original dispersion function before matching.

\section{Comparison of $\delta D^{\prime}$ from model and measurements}

The $\delta D^{\prime}$ from model and measurements are compared for injection $(23.8 \mathrm{GeV})$ and top $(254.8 \mathrm{GeV})$ energies in Table. I. The relative deviations of the measurements from model, ( $\left.\delta D_{\text {measurement }}^{\prime}-\delta D_{\text {model }}^{\prime}\right) / \delta D_{\text {model }}^{\prime}$, without $D^{\prime}$-matching were $10 \%-20 \%$. With $D^{\prime}$-matching, the $\delta D^{\prime}$ were reduced by a factor of $\sim 20$ at injection energy, and by $\sim 500$ at top energy.
The discrepancies between model and measurements in Table I can be explained by the presence of dipole errors and of residual betatron coupling [38].

\section{IMPLEMENTATION OF DISPERSION PRIME MATCHING DURING BEAM ACCELERATION}

To determine if reduced spin tune spread resulting from dispersion prime matching would lead to an improvement in polarization transmission efficiency, the dispersion prime was matched at multiple intermediate energies along the acceleration cycle. In past polarized proton experiments, approximately $10 \%$ relative spin polarization loss was
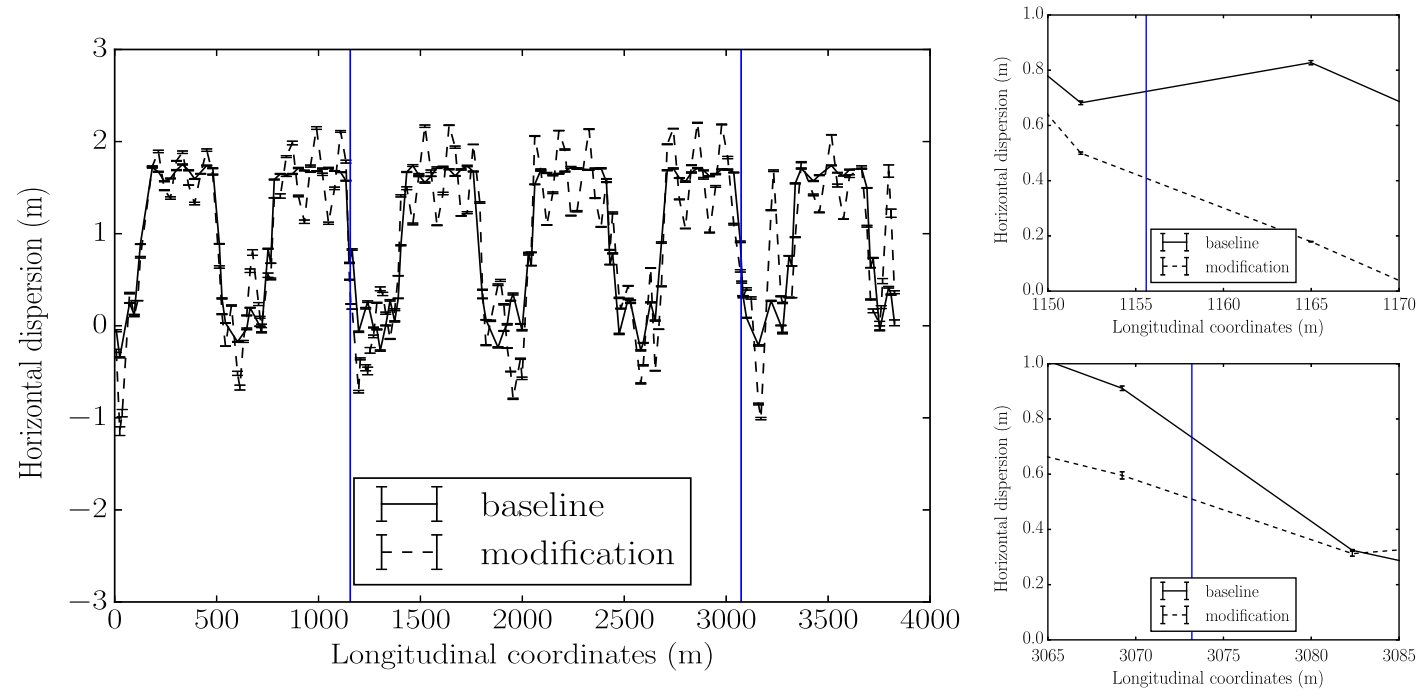

FIG. 6. Left plot: The measured horizontal dispersion functions at the beam position monitors at top energy before (the solid line) and after (the dashed line) the matching of the dispersion primes at the two Siberian snakes (located at the vertical lines) in the blue ring. Right plots: Expanded view of the dispersion functions at the first snake (the upper plot) and the second snake (the lower plot). 
TABLE II. The transmission efficiencies of spin polarization during beam acceleration in the 2017 polarized proton program.

\begin{tabular}{lcccc}
\hline \hline$\epsilon_{\mathrm{BH}}$ & $\epsilon_{\mathrm{BV}}$ & $\epsilon_{\mathrm{YH}}$ & $\epsilon_{\mathrm{YV}}$ & Notes \\
\hline $0.954+/-0.005$ & $0.979+/-0.006$ & $0.915+/-0.005$ & $0.905+/-0.012$ & without $D^{\prime}$-matching \\
$1.020+/-0.004$ & $0.963+/-0.005$ & $0.947+/-0.004$ & $0.953+/-0.010$ & with $D^{\prime}$-matching in blue only \\
\hline \hline
\end{tabular}

observed during acceleration to top energy [36,39]. During the latter part of the 2017 polarized proton program, the dispersion matching scheme for acceleration was applied in the blue ring. The polarization transmission efficiency over the time period with $D^{\prime}$-matching was compared with an earlier period without $D^{\prime}$-matching. The time-averaged transmission efficiencies of spin polarization, defined as the ratio of spin polarization at top and injection energy, were measured. $\epsilon_{\mathrm{BH}}, \epsilon_{\mathrm{BV}}\left(\epsilon_{\mathrm{YH}}, \epsilon_{\mathrm{YV}}\right)$ are the transmission efficiencies measured by the horizontal and vertical Carbon ribbon targets of the blue (yellow) ring $\mathrm{p}$-Carbon polarimeters [40], each consisting of $6 \mathrm{Si}$ strip detectors arranged horizontally and diagonally in the plane transverse to the beam direction, measuring asymmetries of scattered protons from the targets.

The transmission efficiencies of spin polarization in both rings for the same time periods are listed in Table II for comparison. The average transmission efficiency of spin polarization in the blue ring, the average of columns 1 and 2 , was higher by $2.5 \%$ with the dispersion primes matched. However, the transmission efficiency of spin polarization in the yellow ring, the average of columns 3 and 4, was higher by $4.0 \%$ without $D^{\prime}$-matching applied. The improvement of the transmission efficiency in the two rings could not be attributed to any discernible difference in beam emittances. Therefore, the improvement of the polarization transmission efficiency due to reduced spin tune spread was not conclusively proven. This result may indicate that the polarization transmission efficiency has other dependencies, such as deviation from $\nu_{s p}=1 / 2$, snake resonances [41] or interference between intrinsic and imperfection resonances [42]. Simulation and experimental studies are planned to further investigate the effect of spin tune spread on the transmission efficiency of spin polarization during beam acceleration at RHIC.

\section{SUMMARY}

A way to reduce the spin tune spread by matching the dispersion primes at the two Siberian snakes has been developed and implemented for RHIC. The $\gamma_{T}$ quadrupoles, designed for crossing transition energy during beam acceleration, were repurposed to match the dispersion primes at the two Siberian snakes with minimal impact on the global optics.

With implementation of the matching, the difference in dispersion primes at the two Siberian snakes $\left(\delta D^{\prime}\right)$ was reduced by a factor of $\sim 20$ at injection energy and $\sim 500$ at top energy. Immediately after implementation of the matching, a significant reduction of the spin tune spread was experimentally verified [10]. As a result, a spin flipping efficiency of $\sim 97 \%$ was achieved at both injection and top energies [10]. Dispersion prime matching was also implemented during beam acceleration. The polarization transmission efficiency was improved by $2.5 \%$ in the accelerator to which the $D^{\prime}$-matching was implemented, however, the narrower spin tune spread was certainly not the sole contribution to the improvement.

In the future, both spin tracking simulations [43] and experimental studies will continue to further explore the benefits of a narrower spin tune spread for optimally preserving proton polarization and maximizing polarization transmission efficiency during acceleration. To date, the sensitivity of spin flipping efficiency to the difference of $D^{\prime}$ at the two Siberian snakes have been confirmed by spin tracking simulations [34]. In addition, the simulations provided guidance on optimization of the sweep time [43]. Spin tracking studies analyzing the effects of a narrower spin tune spread on polarization preservation during beam acceleration are ongoing [43]. Detailed comparisons of experimental results and simulations will be reported separately.

\section{ACKNOWLEDGMENTS}

The authors would like to thank D. Bruno, A. Marusic, and G. Robert-Demolaize for assistance with the implementation of the optics modifications, V. Ranjbar for his insight in polarization preservation during beam acceleration, F. Meot for guidance on spin flip sweep time, and M. Blaskiewicz, W. Fischer, and T. Roser for their encouragement and suggestions to the work. This research was supported by Brookhaven Science Associates, LLC under Contract No. DE-SC0012704 with the U.S. Department of Energy.

[1] S. D. Bass, The spin structure of the proton, Rev. Mod. Phys. 77, 1257 (2005).

[2] I. Alekseev et al., Polarized proton collider at RHIC, Nucl. Instrum. Methods Phys. Res., Sect. A 499, 392 (2003).

[3] T. Roser, Polarized proton acceleration in AGS and RHIC, AIP Conf. Proc. 980, 15 (2008).

[4] M. Bai, A. U. Luccio, W. W. MacKay, V. Ranjbar, and T. Roser, RHIC spin flipper commissioning, AIP Conf. Proc. 675, 771 (2003). 
[5] M. Bai et al., RHIC spin flipper, in Proceedings of the 22nd Particle Accelerator Conference, PAC-2007, Albuquerque, NM (IEEE, New York, 2007), pp. 18471849.

[6] M. Bai et al., Commissioning of RHIC spin flipper, in Proceedings of the International Particle Accelerator Conference, Kyoto, Japan (ICR, Kyoto, 2010), pp. 12241226.

[7] M. Bai et al., RHIC spin flipper status and simulation studies, in Proceedings of the 24th Particle Accelerator Conference, PAC-2011, New York, 2011 (IEEE, New York, 2011), pp. 447-449.

[8] M. Bai et al., RHIC spin flipper commissioning results, in Proceedings of the 3rd International Particle Accelerator Conference, New Orleans, LA, 2012 (IEEE, Piscataway, NJ, 2012), pp. 1302-1304.

[9] Y.S. Derbenev and A. M. Kondratenko, Radiative polarization: Obtaining, control, using, Part. Accel. 8, 115 (1978).

[10] H. Huang, J. Kewisch, C. Liu, A. Marusic, W. Meng, F. Méot, P. Oddo, V. Ptitsyn, V. Ranjbar, and T. Roser, High Spin-Flip Efficiency at $255 \mathrm{GeV}$ for Polarized Protons in a Ring with Two Full Siberian Snakes, Phys. Rev. Lett. 120, 264804 (2018).

[11] M. Bai, V. Ptitsyn, and T. Roser, Impact on spin tune from horizontal orbital angle between snakes and orbital angle between spin rotators, Technical Report No. C-A/AP/334, 2008.

[12] V. Ptitsyn, M. Bai, and T. Roser, Spin tune dependence on closed orbit in RHIC, in Proceedings of the International Particle Accelerator Conference, Kyoto, Japan (ICR, Kyoto, 2010), pp. 4641-4643.

[13] C. Liu et al., Reducing spin tune spread by matching DX prime at Snakes in RHIC, in Proceedings of the 4th International Particle Accelerator Conference, IPAC2013, Shanghai, China, 2013 (JACoW, Shanghai, China, 2013), pp. 2030-2032.

[14] J. Kewisch et al., Correction of the spin chromaticity in RHIC, Technical report No. C-A/AP/478, 2013.

[15] A. Zelenski et al., Optically pumped polarized $\mathrm{H}$-ion source for RHIC spin physics, Rev. Sci. Instrum. 73, 888 (2002).

[16] M. Harrison, T. Ludlam, and S. Ozaki, RHIC project overview, Nucl. Instrum. Methods Phys. Res., Sect. A 499, 235 (2003).

[17] E. D. Courant and R. D. Ruth, Acceleration of polarized protons in circular accelerators, BNL Technical Report No. 51270, 1980.

[18] S.-Y. Lee, Spin Dynamics and Snakes in Synchrotrons (World Scientific Publishing, Singapore, 1997).

[19] F. Z. Khiari et al., Acceleration of polarized protons to $22 \mathrm{GeV} / \mathrm{c}$ and the measurement of spin-spin effects in $p_{\uparrow}+p_{\uparrow} \rightarrow \mathrm{p}+\mathrm{p}$, Phys. Rev. D 39, 45 (1989).

[20] A. U. Luccio, Computer study of harmonic orbit correction in the AGS Booster, BNL Technical Report No. BNL105175-2014-TECH, 1988.

[21] T. Roser, Properties of partially excited Siberian snakes, AIP Conf. Proc. 187, 1442 (1989).
[22] H. Huang et al., Preservation of Proton Polarization by a Partial Siberian Snake, Phys. Rev. Lett. 73, 2982 (1994).

[23] T. Roser et al., Helical partial snake for the AGS, Technical Report No. AGS/RHIC/SN 072, 1998.

[24] H. Huang et al., Overcoming Depolarizing Resonances with Dual Helical Partial Siberian Snakes, Phys. Rev. Lett. 99, 154801 (2007).

[25] F. Lin et al., Exploration of horizontal intrinsic spin resonances with two partial Siberian snakes, Phys. Rev. ST Accel. Beams 10, 044001 (2007).

[26] V. Schoefer et al., Increasing the AGS beam polarization with 80 tune jumps, in Proceedings of the 3rd International Particle Accelerator Conference, New Orleans, LA, 2012 (IEEE, Piscataway, NJ, 2012), pp. 1015-1019.

[27] H. Huang et al., Overcoming horizontal depolarizing resonances with multiple tune jumps, Phys. Rev. ST Accel. Beams 17, 081001 (2014).

[28] V. Ptitsyn and Y. M. Shatunov, Helical spin rotators and snakes, Nucl. Instrum. Methods Phys. Res., Sect. A 398, 126 (1997).

[29] B. B. Blinov et al., Spin Flipping in the Presence of a Full Siberian Snake, Phys. Rev. Lett. 81, 2906 (1998).

[30] B. B. Blinov et al., 99.6\% Spin-Flip Efficiency in the Presence of a Strong Siberian Snake, Phys. Rev. Lett. 88, 014801 (2001).

[31] V. A. Anferov, B. B. Blinov, D. Yu. Kantsyrev, A. D. Krisch, D. W. Sivers, C. M. Chu, P. Schwandt, B. von Przewoski, and V.N. Grishin, Spin flipping a stored polarized proton beam with an rf dipole, Phys. Rev. ST Accel. Beams 3, 041001 (2000).

[32] M. A. Leonova et al., Achieving 99.9\% Proton Spin-Flip Efficiency at Higher Energy with a Small RF Dipole, Phys. Rev. Lett. 93, 224801 (2004).

[33] M. Bai and T. Roser, Full spin flipping in the presence of full Siberian snake, Phys. Rev. ST Accel. Beams 11, 091001 (2008).

[34] F. Meot et al., RHIC spin flipper, fast-sweep efficiency simulations, Technical Report No. C-A/AP/ 589, 2017.

[35] H. Grote and F. Schmidt, MAD-X - an upgrade from MAD8, in Proceedings of the 20th Particle Accelerator Conference, PAC-2003, Portland, OR, 2003 (IEEE, New York, 2003), pp. 3497-3499.

[36] V. Ranjbar et al., RHIC polarized proton operation for 2017, in Proceedings of IPAC2017, pp. 2188-2190, https://doi.org/10.18429/JACoW-IPAC2017-TUPVA050.

[37] A. L. Gerasimov, D. N. Shatilov, and A. A. Zholents, Beam-beam effects with large dispersion function at the interaction point, Nucl. Instrum. Methods Phys. Res., Sect. A 305, 25 (1991).

[38] C. Liu, Y. Luo, and M. Minty, Simultaneous global coupling and vertical dispersion correction in RHIC, Nucl. Instrum. Methods Phys. Res., Sect. A 750, 56 (2014).

[39] V. Ranjbar et al., RHIC polarized proton operation for 2013, in Proceedings of the 4th International Particle Accelerator Conference, IPAC-2013, Shanghai, 
China, 2013 (JACoW, Shanghai, China, 2013), pp. 15441546.

[40] H. Huang et al., Operation experience of p-Carbon polarimeter in RHIC, in Proceedings of IPAC2015 (2015), pp. 956-958, https://doi.org/10.18429/JACoWIPAC2015-MOPTY011.

[41] V. H. Ranjbar, S. Y. Lee, H. Huang, A. U. Luccio, W. W. MacKay, V. Ptitsyn, T. Roser, and S. Tepikian, Observation of Higher-Order Snake Resonances in Polarized Proton Acceleration in RHIC, Phys. Rev. Lett. 91, 034801 (2003).

[42] V. H. Ranjbar, F. Méot, M. Bai, D. T. Abell, and D. Meiser, Polarization response of RHIC electron lens lattices, Phys. Rev. Accel. Beams 19, 101002 (2016).

[43] F. Meot (private communication). 